\title{
Análisis Experimental del Rendimiento Térmico, Potencia y Emisiones de Cocinas Anafe a Gas Licuado de Petróleo para Altitudes entre 2200 Y 4200 metros
}

\author{
Freddy J. Rojas, Fernando O. Jiménez, Bryan G. Ramos, Renzo Alván \\ Facultad de Ingeniería, Sec. Ing. Mecánica, Pontificia Universidad Católica del Perú, Av. \\ Universitaria 1801, San Miguel, Lima-Perú (e-mail: fjrojas@pucp.edu.pe; ojimene@pucp.edu.pe; \\ bgramos@pucp.pe; ralvan@pucp.pe)
}

Recibido Dic. 7, 2016; Aceptado Feb. 8, 2017; Versión final Abr. 7, 2017, Publicado Ago. 2017

\begin{abstract}
Resumen
Se presentan los resultados del análisis experimental de rendimiento térmico, potencia y emisiones de cocinas a gas licuado de petróleo a altitudes de 2200 a 4200 metros en Perú. Se escogieron cinco localidades representativas entre estas altitudes. Se utilizó un módulo diseñado, e implementado para la toma de datos y se usaron como referencia normas técnicas internacionales para el procedimiento de cálculo de la eficiencia térmica, potencia y emisiones. Los resultados muestran que la cocina prototipo supera altamente en los tres aspectos a las cocinas comerciales. Se concluye que la potencia, emisiones y rendimiento térmico varían conforme a la altitud sobre el nivel del mar, lo cual evidencia que en el diseño de las cocinas comerciales no fue contemplada una variación de altitud. También, se observó que mediante una cocina mejorada (prototipo) es posible conseguir un rendimiento térmico mayor a $50 \%$ y emisiones por debajo de $0.15 \%(\mathrm{CO})_{\mathrm{N}}$.
\end{abstract}

Palabras Clave: gas licuado de petróleo; rendimiento térmico, potencia y emisiones en altitud; cocina anafe

\section{Experimental Analysis of Thermal Performance, Power and Emissions of Liquid Petroleum Gas Stoves at Altitudes between 2200 and 4200 meters}

\begin{abstract}
The results of an experimental analysis of thermal efficiency, power and emissions of liquefied petroleum gas stoves at altitudes between of 2200 and 4200 meters in Peru are presented. Five representative locations between these altitudes were chosen. A module designed and implemented for data collection was employed and reference international technical standards were used for calculating thermal efficiency, power and emissions. The results show that the prototype stove highly exceeds the three aspects compared to commercial stoves. It is concluded that power, thermal efficiency and emissions vary according to the altitude above sea level, which shows that in designing commercial stoves altitude was not considered. It was also observed that using the proposed stove prototype it is possible to achieve thermal efficiency higher than $50 \%$ and emissions below $0.15 \%(\mathrm{CO})_{\mathrm{N}}$.
\end{abstract}

Keywords: liquid petroleum gas; thermal performance, power and emissions in altitude; stove 


\section{INTRODUCCIÓN}

El gas licuado de petróleo (GLP) se ha convertido en uno de los principales combustibles para las familias en el Perú. Según información del Ministerio de Energía y Minas, durante los años 2000 y 2010 la demanda se ha triplicado respecto a años anteriores. La estructura de la venta de GLP hasta diciembre del 2010, menciona que un $64 \%$ de la misma corresponde a Lima Metropolitana. (Mercado de GLP OSINERGMIN, 2011). Así mismo según este informe el consumo de combustible en las cocinas genera emisiones al ambiente para el caso del usuario residencial que consume 18 cilindros de GLP al año, el volumen de emisiones de dióxido de carbono se estima en 523 kilogramos de $\mathrm{CO}_{2}$ anuales (Dirección General de Electricidad Ministerio de Energía y Minas 2008). A pesar de la relevancia de la cocina como máquina térmica en el hogar, no existe un estudio experimental y normativo de cocinas anafe (cocina doméstica sin horno) utilizando gas licuado de petróleo para altitudes sobre el nivel del mar en el rango de los 2200 y 4200 metros.

Como referencia del trabajo se utiliza la normas técnica chilena Nch927/1 (INN-CHILE, 2001) y la norma técnica ecuatoriana NTE 2259 (INEN, 2000). La primera sugiere un rendimiento térmico por encima de $58 \%$ y emisiones de monóxido de carbono neutro $(\mathrm{CO})_{\mathrm{N}}$ por debajo de $0.1 \%$, considerando para el cálculo el poder calorífico inferior $(\mathrm{PCl})$ del combustible. La segunda sugiere un rendimiento térmico por encima de 48 $\%$ y emisiones de monóxido de carbono neutro $(\mathrm{CO})_{\mathrm{N}}$ por debajo de $0.14 \%$, considerando el $\mathrm{PCl}$ del combustible. Ambas normas solo consideran los ensayos a una altitud a la del nivel del mar. A la fecha del trabajo de investigación realizado no hay una norma técnica nacional ni internacional para evaluar a condiciones de mayores altitudes.

Desde el uso de quemadores domésticos muchos investigadores han estudiado los factores que pueden influir en la mejora de su combustión, y como consecuencia de ello optimizar el rendimiento térmico y disminuir emisiones, factores que influyen en el ahorro y salud del consumidor. Surange et. al., (2014) en su trabajo realiza un consolidado de diferentes investigaciones orientadas a la mejora de quemadores domésticos que utilizan GLP, del cual concluye que factores como el material y diseño del quemador, la geometría del mezclador, así como la relación del diámetro del inyector y los puertos de salida de la cabeza del quemador influyen en la mejora de la combustión. Sin embargo, los estudios mencionados se enfocan a condiciones de operación sobre el nivel del mar. En el mundo se encuentran poblaciones situadas a una altitud en el rango de 1000 - 4800 m.s.n.m. En la mayoría de ellas que se encuentran en zonas remotas, se utiliza como fuente de energía la leña para la cocción o calefacción. En respuesta a ello se estudiaron propuestas para mejorar los niveles de confort en una vivienda situada a gran altitud al noroeste de Nepal (Fuller et al., 2009), propuesta que incluye el uso de una cocina a leña mejorada, de la misma forma Aggarwal \& Chandel (2004) implementaron el sistema de cocinas de leña mejorada en el estado occidental Himalaya de la India. Ambos estudios afirman la necesidad de mejorar estas cocinas en favor de la salud de las personas y del beneficio del medio ambiente.

Respecto a las poblaciones a una altitud mayor a $1000 \mathrm{~m}$ sobre el nivel del mar que utilizan gas combustible (GLP o GN ) como fuente de energía para la cocción de alimentos, Amell et al. (2016) estudiaron el efecto de la potencia en quemadores atmosféricos domésticos a GLP o GN en las localidades de Colombia como Santa de Antioquía (555 m), Medellín (1550 m) y Alto Bogotá (2600 m); del resultado de su estudio afirmaron que la potencia de un quemador atmosférico disminuye $1.5 \%$ por cada $304 \mathrm{~m}$ de incremento de altitud. Por su parte, Zhou et al. (2017) investigaron acerca de la influencia de la altitud en la potencia, rendimiento térmico y emisiones en quemadores atmosféricos domésticos a GN en las localidades Chinas de Lhasa $(3658 \mathrm{~m})$ y Chongqing $(4000 \mathrm{~m})$. El estudio concluye que un cambio en la presión de suministro del gas combustible puede compensar la pérdida de potencia que es producida por el incremento de altitud, el rendimiento térmico incrementa a medida que la altitud aumenta y las emisiones incrementan por la falta de oxígeno en el aire atmosférico a una mayor altitud.

El presente trabajo propone contribuir a la literatura actual con un estudio experimental del rendimiento térmico, potencia y emisiones de cuatro cocinas anafes comerciales y una cocina prototipo diseñada con mejores características de performance utilizando gas licuado de petróleo (GLP) en las localidades cuyas altitudes se encuentran entre 2200 y 4200 metros. Y además, sugerir requerimiento de rendimiento térmico y emisiones para el correcto funcionamiento de quemadores atmosféricos domésticos por encima de los 2000 metros.

\section{MATERIALES Y MÉTODOS}

Se hace una descripción detallada de los equipos e instrumentos usados, luego se describen algunas características del combustible empleado, para finalmente describir los procedimientos usados en los ensayos. 


\section{Equipos e instrumentos}

Se usó cinco cocinas tipo anafe (cocina doméstica sin horno) de una potencia aproximada de $1.5 \mathrm{~kW}$ (a condiciones de altitud de Lima). Una balanza de rango 0 a $20 \mathrm{~kg}$, dos ollas de aluminio de $20 \mathrm{~cm}$ y $22 \mathrm{~cm}$, una campana extractora fabricada según norma técnica internacional (INN-CHILE, 2001) e instrumentación adecuada tal como se presentan en la Tabla 1.

Tabla 1: Instrumentación

\begin{tabular}{|c|c|c|c|}
\hline Instrumento & Rango & Precisión & Condiciones \\
\hline Indicador de temperatura ambiental & -20 a $80{ }^{\circ} \mathrm{C}$ & $\begin{array}{l}+/-0.3^{\circ} \mathrm{C} \\
+/-0.4^{\circ} \mathrm{C}\end{array}$ & $\begin{array}{l}\text { De } 0 \text { a } 70^{\circ} \mathrm{C} \\
\text { Restante }\end{array}$ \\
\hline Indicador de humedad & 0 a 100 & $\begin{array}{l}+/-2.0 \% \\
+/-2.5 \% \\
\end{array}$ & $\begin{array}{l}\text { De } 10 \text { a } 90 \% \mathrm{RH} \\
\text { Restante }\end{array}$ \\
\hline Indicador de presión atmosférica & $\begin{array}{c}600 \text { a } 1100 \\
\text { mbar }\end{array}$ & +/- 0.5 mbar @25 $\mathrm{C}$ & \\
\hline Termocupla tipo $\mathrm{K}$ & 0 a $500^{\circ} \mathrm{C}$ & Norma IEC584-Clase 2 & \\
\hline Medidor de flujo & 0 a 50 SLPM & $\begin{array}{c}+/-0.8 \% \text { de la lectura } \\
+/-0.2 \% \text { full }\end{array}$ & $\begin{array}{l}\text { Max presión de línea: } 145 \text { psi } \\
\text { Temperatura de operación:- } 10 \text { a } 50^{\circ} \mathrm{C}\end{array}$ \\
\hline $\begin{array}{l}\text { Manómetro digital } \\
\text { Analizador de gases (Quintox) }\end{array}$ & -1 a 2 bar & $0.05 \% F E$ & $\begin{array}{l}\text { Resolución: } 0.1 \text { mbar } \\
\text { Temperatura de trabajo: } 0 \text { a } 50^{\circ} \mathrm{C} \\
\text { Incertidumbre: }+/-20 \mathrm{ppm}\end{array}$ \\
\hline
\end{tabular}

Los equipos utilizados fueron un regulador Rego de rango de entrada de 0 a 7 bar y salida máxima de 31 mbar. Las cocinas comerciales y prototipo están descritas en la Tabla 2. Las cocinas comerciales son las que se venden y se usan a nivel nacional, y la cocina prototipo es la cocina diseñada adecuadamente en este proyecto para mejorar la eficiencia térmica.

Tabla 2: Modelos de cocinas comerciales y prototipo a ensayar

\begin{tabular}{|l|l|l|l|}
\hline Código de Cocinas & \multicolumn{1}{|c|}{ Marca } & Material/Recubrimiento & \multicolumn{1}{c|}{ Hornilla } \\
\hline CA-01 & Superior/surge & Acero inoxidable & 2 piezas (dentado) \\
\hline CE-01 & Superior/surge/fadicc & pintado & 2 piezas (dentado) \\
\hline CL-01 & Citecil/fadicc & enlozado & 2 piezas (agujeros) \\
\hline CE-02 & Superior/surge/fadicc & pintado & 1 piezas (agujeros) \\
\hline CP-01 & "Prototipo" & Acero inoxidable & 1-pieza (agujeros) \\
\hline
\end{tabular}

\section{Combustible}

Durante los ensayos se usó el gas licuado de petróleo (GLP), con este combustible se trabajó con una presión de 28 mbar, pues este es el valor de operación común en las instalaciones a nivel residencial. De modo que los valores a utilizar para el cálculo de rendimiento se muestran en la Tabla 3:

Tabla 3: Datos del GLP (Rojas, 2016)

\begin{tabular}{|l|c|c|}
\hline \multicolumn{1}{|c|}{ Variable } & Unidad & Valor \\
\hline Presión manométrica del flujo & $\mathrm{mbar}$ & 28 \\
\hline Caudal & $\mathrm{LPM}$ & 1.08 \\
\hline Poder Calorífico Inferior & $\mathrm{kJ} / \mathrm{kg}$ & 46090 \\
\hline Densidad & $\mathrm{kg} / \mathrm{m}^{3}$ & 2.1023 \\
\hline Relación de calores específicos $(\mathrm{k})$ & - & 1.135 \\
\hline Densidad Relativa $(\mathrm{s})$ & - & 1.7161 \\
\hline Peso Molecular & $\mathrm{Kg} / \mathrm{kmol}$ & $17.7 \times 10^{-3}$ \\
\hline
\end{tabular}




\section{Metodología}

Para el trabajo de campo se ha organizado un equipo de viaje a cinco ciudades cuyas altitudes están en el rango de 2000 y 4200 metros. Matucana, Tarma, Huancayo, La Oroya y Cerro Pasco. En la tabla 3 se muestra las altitudes para las que fueron realizados los ensayos.

Tabla 3: Localidades y altitudes de ensayo

\begin{tabular}{|l|c|}
\hline \multicolumn{1}{|c|}{ Localidad } & Altitud de ensayo (metros sobre el nivel del mar) \\
\hline Matucana & 2293 \\
\hline Tarma & 2891 \\
\hline Huancayo & 3126 \\
\hline La Oroya & 3706 \\
\hline Cerro de Pasco & 4155 \\
\hline
\end{tabular}

Para los ensayos de emisiones y rendimiento térmico para quemadores atmosféricos (cuyo diseño fue realizado mediante modelos matemáticos según Baukal, 2003; Fulford, 1996; H.R.N. Jones, 2005) se realizaron en base a la norma técnica chilena Nch927/1. El número de repeticiones que se realizó para ensayos de eficiencia térmica y emisiones de $\mathrm{CO}$ fueron 3 veces. Es así que, siguiendo con lo estipulado por la norma NCh927/1, se ha de utilizar dos recipientes, cuyas dimensiones se encuentran ya establecidas, para calentar el agua. El primero es una olla hecha de aluminio con $20 \mathrm{~cm}$ de diámetro, esta contendrá 3.7 $\mathrm{kg}$ de agua y su función es la de precalentar el quemador antes de comenzar con los ensayos. El segundo es otra olla hecha de aluminio de $22 \mathrm{~cm}$ de diámetro, con la cual se realizaron las experiencias (INN-CHILE, 2001). La Tabla 4 muestra los valores de las variables para los ensayos. Mientras que el proceso para el análisis de la emisión de monóxido de carbono neutro $\left(\mathrm{CO}_{n}\right)$ consiste en hacer uso de una olla de $22 \mathrm{~cm}$ con $3,7 \mathrm{~kg}$ de agua y una campana extractora que posea las dimensiones indicadas en la norma NCh 927/1 (INN-CHILE, 2001).

Tabla 4: Valores de las variables para los ensayos

\begin{tabular}{|l|c|c|}
\hline \multicolumn{1}{|c|}{ Variable } & Unidad & Valor \\
\hline Masa de agua & $\mathrm{kg}$ & 3.7 \\
\hline Calor específico del agua & $\mathrm{kJ} / \mathrm{kg}{ }^{\circ} \mathrm{C}$ & 4.186 \\
\hline Temperatura inicial del agua & ${ }^{\circ} \mathrm{C}$ & 25 \\
\hline Temperatura final del agua & ${ }^{\circ} \mathrm{C}$ & 90 \\
\hline Temperatura del gas & ${ }^{\circ} \mathrm{C}$ & 21 \\
\hline
\end{tabular}

Para dar inicio a los ensayos se tomó nota de las condiciones ambientales el lugar, así mismo se registró la temperatura a la cual bulle el agua; debido a que cambia por la presión atmosférica del lugar de ensayo (Cengel, 2003). De esta manera se escoge el rango de temperaturas para el ensayo de rendimiento térmico, por lo general se tomó según se indica en la Tabla 4. Para el precalentamiento del sistema y posterior análisis de gases se introduce una masa de $3.7 \mathrm{~kg}$ de agua en un recipiente de aluminio de $22 \mathrm{~cm}$ de diámetro mediante el uso de una balanza.

Luego de que el agua llegue a bullir se sitúa la campana sobre el recipiente para lo toma de emisiones con un analizador de gases. Se coloca la varilla de medición, la inestabilidad de las emisiones debe de ser la mínima, los valores de ppm de monóxido de carbono (CO) y la relación de $\mathrm{CO}$ y $\mathrm{CO}_{2}$ deben ser casi constantes, para ello se deja un tiempo adecuado para alcanzar el cuasi-equilibrio y luego se registra en el equipo, tal como se muestra en la Figura 1.

Una vez registrada la relación $\frac{(\mathrm{CO})_{\mathrm{M}}}{\left(\mathrm{CO}_{2}\right)_{\mathrm{M}}}$ con el analizador de gases y usando la ecuación (1), el porcentaje de monóxido de carbono calculado $\left(\%(\mathrm{CO})_{\mathrm{N}}\right)$ es obtenido de la norma (INN-CHILE, 2001, pág. 46)

$\%(\mathrm{CO})_{\mathrm{N}}=\%\left(\mathrm{CO}_{2}\right)_{\mathrm{N}} * \frac{(\mathrm{CO})_{\mathrm{M}}}{\left(\mathrm{CO}_{2}\right)_{\mathrm{M}}}$

Donde $\%(\mathrm{CO})_{\mathrm{N}}$ es el porcentaje de $\mathrm{CO}$ referido a los productos de combustión privados de aire y vapor de agua; \% $\left(\mathrm{CO}_{2}\right)_{\mathrm{N}}$ es el porcentaje de $\mathrm{CO}_{2}$ calculado para los productos de la combustión del gas considerado, exentos de aire y de vapor de agua; y $(\mathrm{CO})_{\mathrm{M}}$ y $\left(\mathrm{CO}_{2}\right)_{\mathrm{M}}$ son las concentraciones de monóxido de carbono y dióxido de carbono medidas en las muestras tomadas durante el ensayo de combustión, expresadas en las mismas unidades. 


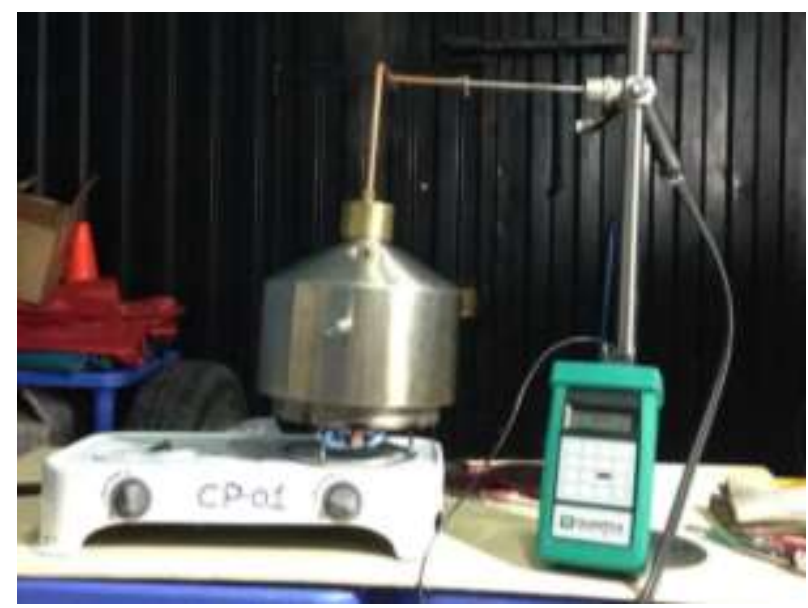

Fig. 1: Prueba de emisiones mediante el analizador de gases

El porcentaje hallado se compara con los máximos permisibles, según norma para nuestras condiciones de trabajo los quemadores no deben emitir más de $0.1 \%$ de CO (INN-CHILE, 2001, pág.24). Para hallar el rendimiento térmico, se debe medir el flujo de trabajo en LPM y para hallar el flujo estándar se calculó por medio de la ecuación (2). Luego de ello se halla la potencia en función del PCl, y luego por medio de la Tabla 5 (INN-CHILE, 2001, pág. 47) se escoge el recipiente adecuado para el ensayo de rendimiento.

$\mathrm{V}_{\mathrm{SLPM}}=* \mathrm{~V}_{\mathrm{LPM}} * \frac{298}{\mathrm{~T}_{\mathrm{en}}} * \frac{\mathrm{P}_{\mathrm{en}}}{101,325}$

Donde, $\mathrm{V}_{\mathrm{SLPM}}$ es el flujo volumétrico corregido estándar, (SLPM); $\mathrm{V}_{\mathrm{LPM}}$ es el flujo volumétrico medido durante el ensayo, (LPM); $\mathrm{P}_{\mathrm{en}}$ es la presión absoluta durante el ensayo, $(\mathrm{kPa}) ; \mathrm{y} \mathrm{T}_{\mathrm{en}}$ es la Temperatura medida durante el ensayo, $(\mathrm{K})$

Tabla 5: Diámetro de recipiente, masa de agua y consumos

\begin{tabular}{|c|c|c|c|}
\hline Diámetro interior, $\mathrm{mm}$ & Consumo con PCS, $\mathrm{kW}$ & Consumo con $\mathrm{PCl}, \mathrm{kW}$ & Masa de agua, $\mathrm{kg}$ \\
\hline 160 & 1.05 & 0.94 & 1.4 \\
\hline 180 & 1.33 & 1.20 & 2.0 \\
\hline 200 & 1.65 & 1.48 & 2.8 \\
\hline 220 & 1.99 & 1.79 & 3.7 \\
\hline 240 & 2.37 & 2.13 & 4.8 \\
\hline 260 & 2.78 & 2.50 & 6.1 \\
\hline 280 & 3.22 & 2.90 & 7.7 \\
\hline 300 & 3.70 & 3.33 & 9.4 \\
\hline 320 & 4.20 & 3.78 & 11.4 \\
\hline
\end{tabular}

Se coloca la olla sobre la parrilla de la cocina con el quemador encendido luego se introduce la Termocupla tipo $\mathrm{K}$ dentro de la olla, aproximadamente a la mitad de la altura del volumen de agua, como se indica en la Figura 2. Se toma el tiempo para el cual la temperatura cubre el rango de trabajo para hallar el volumen consumido de combustible.

Los datos obtenidos son utilizados para calcular el rendimiento térmico del quemador como indica la ecuación (3) en la norma (INN-CHILE Modificada 2001, 48).La cual menciona que para las condiciones de ensayo definidas el rendimiento térmico debe ser igual o superior a $52 \%$ con PCS y $58 \%$ con PCl.

$\mathrm{R}=\mathrm{M} * \mathrm{C}_{\mathrm{P}} * \frac{\mathrm{t}_{2}-\mathrm{t}_{1}}{\mathrm{~V} * \mathrm{PC}} * 100$

Donde, $\mathrm{R}$ es el rendimiento, \%.; $\mathrm{M}$ es la masa de agua introducida en el recipiente, $\mathrm{kg}$; $\mathrm{C}_{\mathrm{p}}$ es $4.186^{*} 103$ $\left(\mathrm{J} / \mathrm{kg}^{\circ} \mathrm{C}\right) ; \mathrm{T}_{1}$ es la temperatura inicial del agua, ${ }^{\circ} \mathrm{C} ; \mathrm{T}_{2}$ es la temperatura máxima del agua después de la extinción, en ${ }^{\circ} \mathrm{C}$; $\mathrm{V}$ es la volumen o masa de gas consumido en $\mathrm{m}^{3}$ (referido a las condiciones de referencia del poder calorífico). 


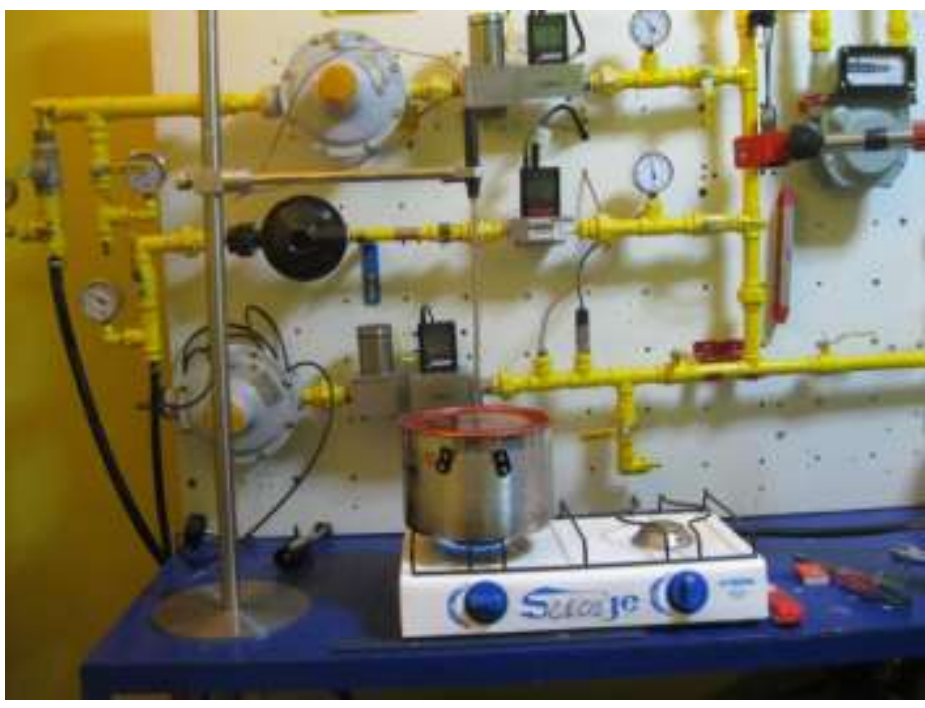

Fig. 2: Banco de ensayo para obtener el Rendimiento Térmico de la cocina a ensayar

\section{RESULTADOS Y DISCUSIÓN}

Los resultados de los ensayos de rendimiento térmico y emisiones son analizados para las cocinas CE-01, CE-02, CL-01, CA-01 tal como fueron fabricados. La cocina prototipo (CP-01) fue ensayada con dos inyectores y hasta tres presiones de trabajo diferentes (28 y 30 mbar) (Tabla 6 y Tabla 7). Además, se presenta en la Tabla 8 el cálculo de la potencia en altitud, variable muy importante para el análisis de resultados.

Tabla 1: Rendimiento térmico (\%) de cocinas a GLP

\begin{tabular}{|c|c|c|c|c|c|c|c|}
\hline Cocina & Presión del gas & Diámetro del inyector & \multirow{2}{*}{ Matucana } & \multirow{2}{*}{ Tarma } & \multirow{2}{*}{ Huancayo } & \multirow{2}{*}{ La Oroya } & \multirow{2}{*}{ Cerro de Pasco } \\
\hline Código & mbar & $\mathrm{mm}$ & & & & & \\
\hline CE-01 & 28 & fábrica & 51.4 & 55.6 & 55.0 & 54.8 & 53.7 \\
\hline CE-02 & 28 & fábrica & 37.3 & 53.9 & 57.8 & 48.7 & 48.0 \\
\hline CL-01 & 28 & fábrica & 38.5 & 43.3 & 60.4 & 55.5 & 51.9 \\
\hline $\mathrm{CA}-01$ & 28 & fábrica & 46.1 & 51.3 & 50.2 & 51.4 & 47.7 \\
\hline CP-01 & 28 & 0.65 & 40.1 & 54.9 & 59.1 & 55.5 & 54.3 \\
\hline CP-01 & 30 & 0.65 & 43.6 & 55.9 & 59.5 & 53.6 & 52.9 \\
\hline CP-01 & 28 & 0.7 & 49.0 & 54.6 & 50.4 & 58.2 & 54.3 \\
\hline CP-01 & 30 & 0.7 & 51.0 & 53.3 & 50.1 & 56.3 & 53.3 \\
\hline
\end{tabular}

Tabla 2: Emisiones (\% monóxido de carbono neutro) de cocinas a GLP

\begin{tabular}{|c|c|c|c|c|c|c|c|}
\hline Cocina & Presión del gas & Diámetro del inyector & \multirow{2}{*}{ Matucana } & \multirow{2}{*}{ Tarma } & \multirow{2}{*}{ Huancayo } & \multirow{2}{*}{ La Oroya } & \multirow{2}{*}{ Cerro de Pasco } \\
\hline Código & mbar & $\mathrm{mm}$ & & & & & \\
\hline CE-01 & 28 & fábrica & 0.36 & 0.11 & 0.15 & 0.14 & 0.18 \\
\hline CE-02 & 28 & fábrica & 0.05 & 0.04 & 0.05 & 0.18 & 0.06 \\
\hline CL-01 & 28 & fábrica & 0.43 & 0.25 & 0.41 & 0.44 & 0.28 \\
\hline $\mathrm{CA}-01$ & 28 & fábrica & 0.33 & 0.28 & 0.37 & 0.21 & 0.44 \\
\hline CP-01 & 28 & 0.65 & 0.03 & 0.04 & 0.09 & 0.06 & 0.03 \\
\hline CP-01 & 30 & 0.65 & 0.05 & 0.06 & 0.08 & 0.13 & 0.04 \\
\hline CP-01 & 28 & 0.7 & 0.11 & 0.11 & 0.13 & 0.10 & 0.19 \\
\hline CP-01 & 30 & 0.7 & 0.11 & 0.10 & 0.09 & 0.13 & 0.08 \\
\hline
\end{tabular}


El análisis de resultados se enfoca en tres propósitos relacionados a los resultados obtenidos, estos son potencia, rendimiento térmico y emisiones. La potencia está ligada al tiempo de funcionamiento del equipo, el rendimiento térmico se traduce en ahorro y las emisiones responden al cuidado de la salud del usuario y el medio ambiente. Para el análisis de los resultados se realiza el análisis energético de la cocina prototipo con el propósito de escoger la configuración óptima para el rango de alturas entre 2200-4200 metros Por último se realiza la comparación de la cocina prototipo óptimo y las cocinas comerciales.

Tabla 8: Potencia $(\mathrm{kW})$ de las cocinas a GLP

\begin{tabular}{|c|c|c|c|c|c|c|c|}
\hline Cocina & Presión del gas & Diámetro del inyector & \multirow{2}{*}{ Matucana } & \multirow{2}{*}{ Tarma } & \multirow{2}{*}{ Huancayo } & \multirow{2}{*}{ La Oroya } & \multirow{2}{*}{$\begin{array}{c}\text { Cerro } \\
\text { de Pasco }\end{array}$} \\
\hline Código & mbar & $\mathrm{mm}$ & & & & & \\
\hline CE-01 & 28 & fábrica & 1.26 & 1.18 & 1.16 & 1.13 & 1.11 \\
\hline CE-02 & 28 & fábrica & 1.16 & 1.07 & 1.05 & 0.90 & 0.91 \\
\hline CL-01 & 28 & fábrica & 1.23 & 1.14 & 1.12 & 1.06 & 1.11 \\
\hline CA-01 & 28 & fábrica & 1.07 & 1.00 & 0.99 & 0.86 & 0.86 \\
\hline CP-01 & 28 & 0.65 & 1.20 & 1.12 & 1.10 & 1.00 & 1.01 \\
\hline CP-01 & 30 & 0.65 & 1.25 & 1.16 & 1.14 & 1.03 & 1.04 \\
\hline CP-01 & 28 & 0.7 & 1.75 & 1.63 & 1.60 & 1.49 & 1.47 \\
\hline CP-01 & 30 & 0.7 & 1.81 & 1.69 & 1.66 & 1.54 & 1.51 \\
\hline
\end{tabular}

\section{Análisis energético de la cocina prototipo}

En las Figuras 3, 4 y 5 se observa los resultados de los ensayos para la cocina prototipo a gas licuado de petróleo con un inyector de $0.65 \mathrm{~mm}$ a 28 y 30 mbar de presión; así como un inyector de $0.7 \mathrm{~mm}$ y presión de trabajo de 28 y 30 mbar. En la Figura 3 se aprecia que el inyector de $0.65 \mathrm{~mm}$ a las presiones de 28 y 30 mbar conforme la altitud se incrementa hasta los 3126 metros incrementa su rendimiento térmico de $52 \%$ a $59 \%$ aproximadamente, las emisiones están por debajo de $0.1 \%(\mathrm{CO})_{\mathrm{N}}$ (Figura 4); sin embargo la disminución de potencia cataloga a la cocina con un diámetro de $0.65 \mathrm{~mm}$ como un quemador semirápido bajo por acercarse a la potencia de $1 \mathrm{~kW}$ a los 3126 metros (Figura 5), por lo que no cumple con el criterio de mejor tiempo de operación.

El diseño de la cocina prototipo está orientado a la operación por encima de los 2000 metros cumpliendo los tres criterios mencionados anteriormente. Debido a esto, se escoge .el inyector de $0.7 \mathrm{~mm}$ por presentar una potencia para un quemador semirápido por encima de los 2000 metros Por lo cual se escoge la presión de 28 mbar por presentar un mayor rendimiento térmico por encima de los 2000 metros. Se concluye que el mejor arreglo para gas licuado de petróleo es con el inyector de $0.7 \mathrm{~mm}$ a una presión de $30 \mathrm{mbar}$.

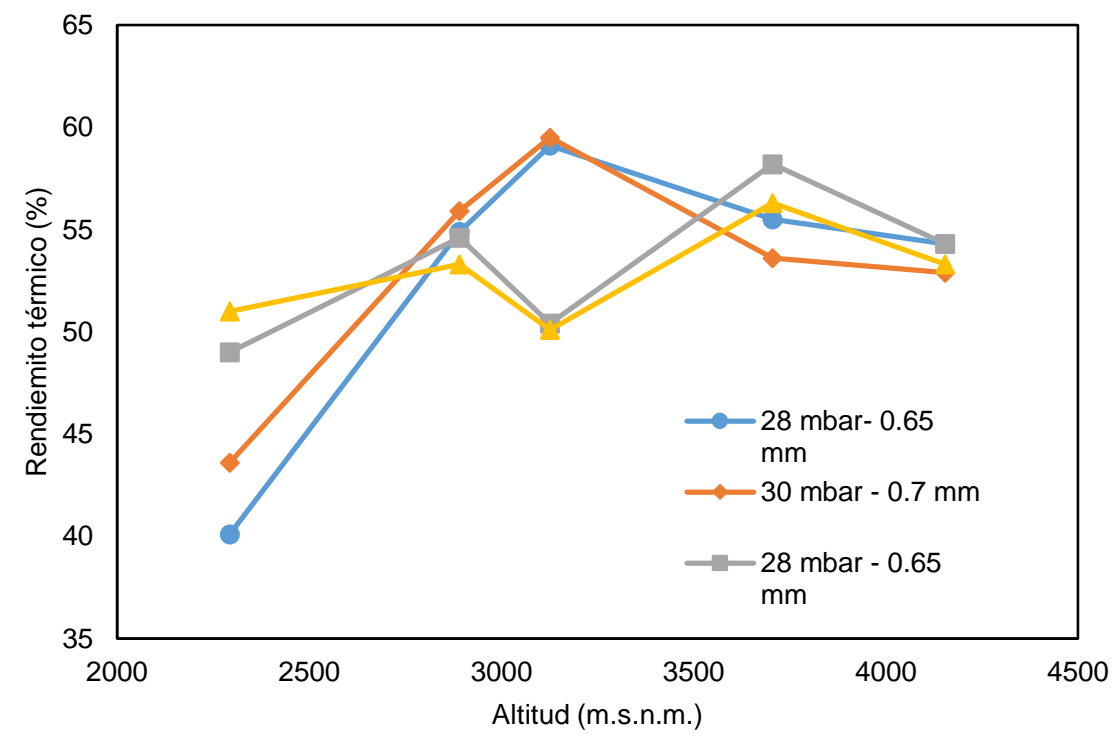

Fig. 3: Rendimiento térmico de la cocina CP-01 a GLP 


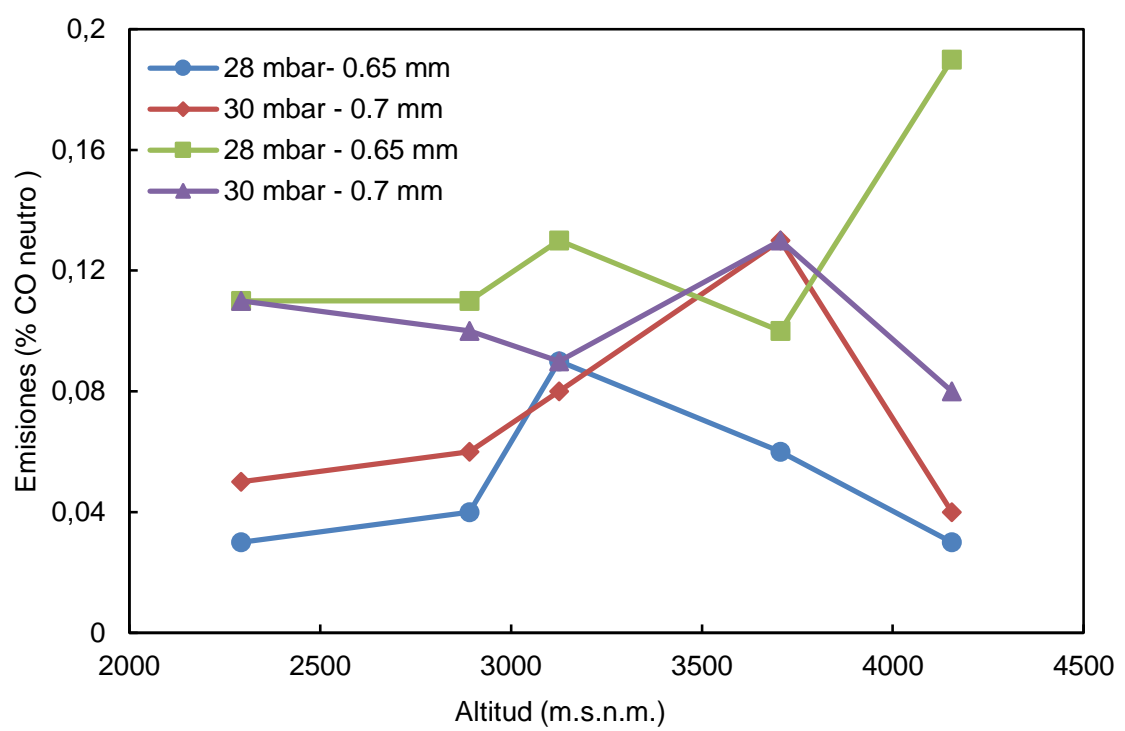

Fig. 4: Emisiones de la cocina CP-01 a GLP

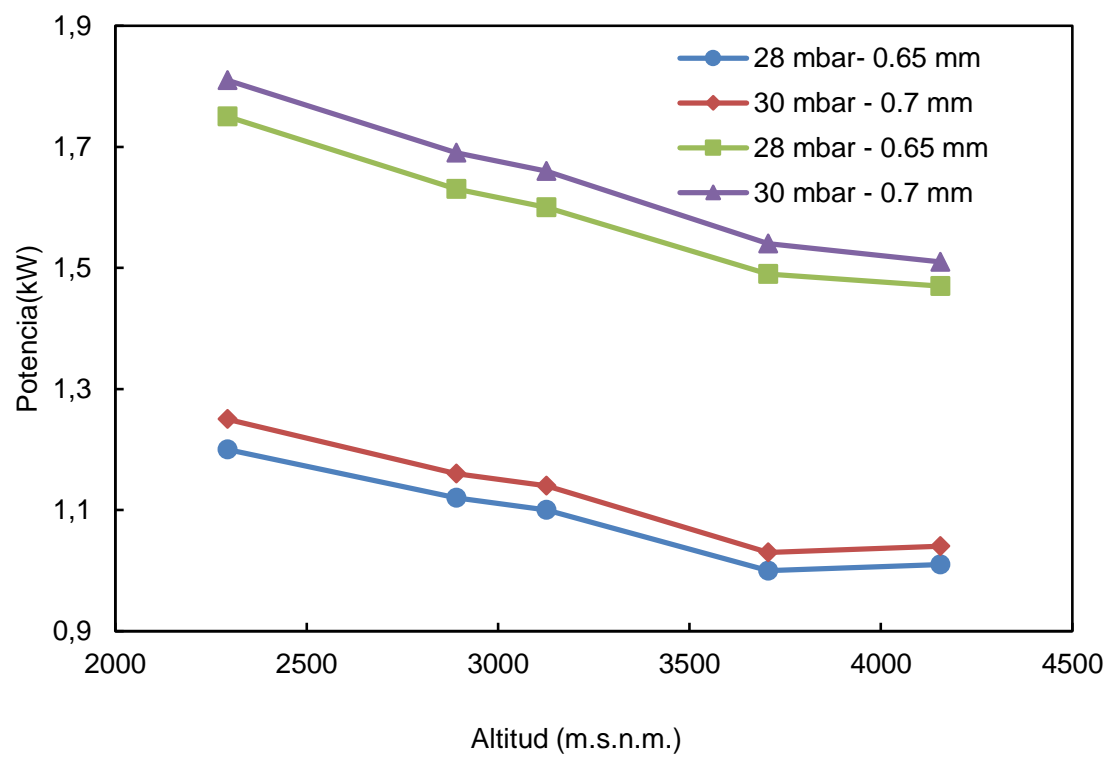

Fig. 5: Potencia de la cocina CP-01 a GLP

\section{Comparación energética de cocinas a GLP}

En la Figura 6, el rendimiento térmico de la cocina prototipo es superior al $50 \%$ a diferencia de las cocinas comerciales, que no superan dicho valor. Además, la cocina prototipo cuenta con valores por debajo de 0.15 $\%(\mathrm{CO})_{\mathrm{N}}$ de emisiones (Figura 7$)$, a diferencia de las cocinas comerciales. Sin embargo a medida que se incrementa la altitud las emisiones disminuyen, lo cual coloca a solo 2 cocinas (CE-02 y CP-01) en un nivel aceptable de emisiones para su uso a partir de los 2000 metros.

En la Figura 8 la potencia es considerablemente mayor en la cocina prototipo (1.90 kW a un altitud de 66 metros) mientras que las comerciales no superan los $1.4 \mathrm{~kW}$ en el mismo punto. Como se mencionó anteriormente, a medida que se aumenta la altitud la potencia disminuye, esto coloca a dos de las cocinas comerciales en las localidades de más de 3000 metros fuera del rango de operación de un quemador semirápido como la cataloga la norma (INN-CHILE Modificada 2001, 15). Finalmente, luego de analizar estos criterios, se concluye que la cocina prototipo cumple con lo necesario, además de lo establecido, para operar mejor sobre los 2000 metros, superando a las cocinas comerciales en potencia, rendimiento térmico y emisiones. 


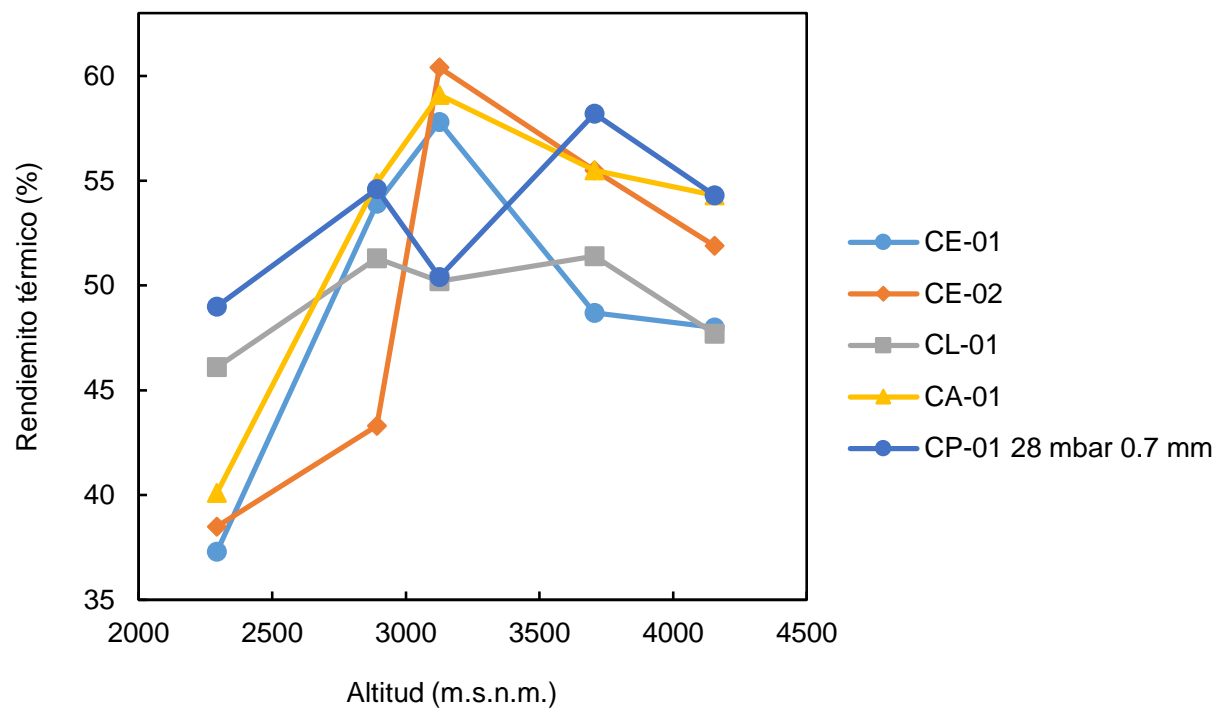

Fig. 6: Rendimiento térmico de las cocinas a GLP

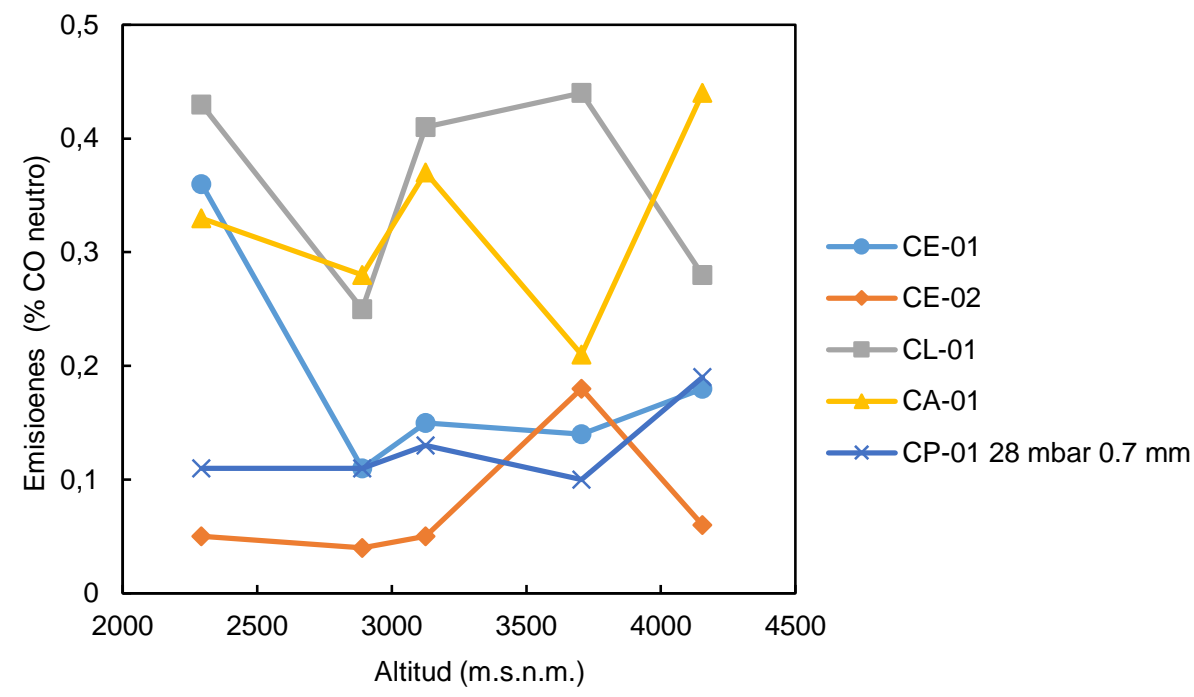

Fig. 7: Emisiones de las cocinas a GLP

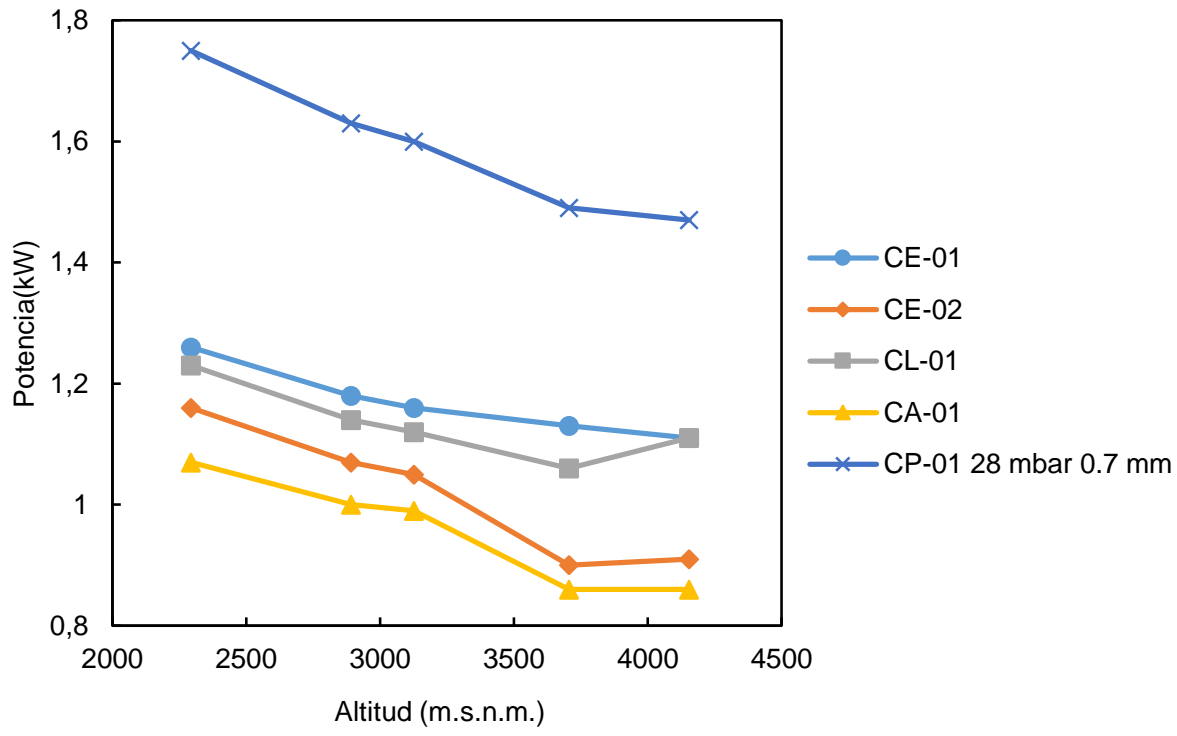

Fig. 8: Potencia de las cocinas a GLP 


\section{DISCUSION FINAL}

En el Perú, no existe una norma técnica referente a rendimiento térmico de cocinas domésticas a gas licuado de petróleo sobre el nivel del mar y aún más, tampoco existe en el Perú y en el mundo una norma técnica para altitud entre los 2200 y 4200 metros por lo cual, este estudio contribuyó a una propuesta de Proyecto de Norma Técnica Peruana (PNTP) nueva. Esta se adjuntó al proyecto INNOVATE PERU que a través de los Proyectos Concursables de Investigación Básica y Aplicada 157-FINCYT-IA-2013 en cooperación con la Pontificia Universidad Católica del Perú se realizó.

La variación de potencia en las cocinas, producto de la variación de la presión atmosférica a medida que se incrementa la altitud sobre el nivel mar, no se contempló en el diseño de las cocinas anafes comerciales. Debido a que se observan variaciones de $1.17 \mathrm{~kW}$ a $0.86 \mathrm{~kW}$ como es en el caso de la cocina CA-01, además se concluye que la cocina pierde su condición de quemador semi - rápido como señalan las normas técnicas de Ecuador y Chile. Por otro lado, mediante el incremento del diámetro del inyector se compensa la perdida de potencia, es así como la cocina prototipo con un inyector de $0.7 \mathrm{~mm}$ a una presión de 28 mbar compensa la pérdida de potencia y opera con una potencia de $1.5 \mathrm{~kW}$, como sería normalmente a una altitud como la de Lima, a una altitud de 4155 metros.

La disminución de potencia con el incremento de la altitud, permite que el rendimiento térmico se incremente como se registró en todas las cocinas anafes ensayados. Sin embargo, a pesar del incremento general en el rendimiento, para una altitud por encima de los 2000 metros la cocina prototipo con un inyector de $0.7 \mathrm{~mm}$ y una presión de 28 mbar tiene un rendimiento térmico superior a $50 \%$ superando de esta manera a 3 de las 4 cocinas comerciales evaluadas.

La cocina CE-02 y CP-01, a una presión manométrica de 28 mbar y $0.7 \mathrm{~mm}$ de diámetro de inyector por encima de los 2000 metros tienen emisiones por debajo de $0.15 \%(\mathrm{CO})_{\mathrm{N}}$. Por lo tanto, la cocina comercial considera en su fabricación este parámetro. La cocina CA-01 y CL-01 por encima de los 2000 metros registran emisiones por encima de $0.3 \%(\mathrm{CO})_{\mathrm{N}}$, debido a que sus rendimientos son por debajo de $50 \%$, convirtiendo el carbono del combustible en $\mathrm{CO}$ lo que nos lleva a concluir que los fabricantes no consideran este parámetro en la fabricación de sus cocinas. Las emisiones así como la potencia y el rendimiento térmico también cambian con el incremento de la altitud por encima de los 2000 metros. Se concluye que un quemador atmosférico doméstico cumplirá satisfactoriamente respecto a tiempo, ahorro y salud si posee un rendimiento térmico mayor a $50 \%$ y emisiones por debajo de $0.15 \%(\mathrm{CO})_{\mathrm{N}}$ por encima de los 2200 metros.

\section{CONCLUSIONES}

De acuerdo a los resultados y su análisis, se pueden extraer las siguientes conclusiones principales de este trabajo:

La metodología planteada para el estudio experimental de las cuatro cocinas anafe comerciales y cocina prototipo utilizando gas licuado de petróleo para alturas entre 2200 y 4200 m.s.n.m. nos permitió obtener la data de condiciones ambientales (presión, temperatura y humedad ), presión manométrica del gas a la entrada de la cocina, flujo volumétrico del gas a las condiciones de ensayo, temperatura inicial y final del agua de calentamiento, masa de agua de calentamiento y tiempo requerido para cumplir con el delta de temperatura. A su vez estos datos permitieron el análisis de potencia, rendimiento térmico y emisiones para cada configuración de estudio, siendo el tiempo aproximado de ensayos por cocina de 1 hora gracias a la metodología planteada.

La potencia de la cocina anafe disminuye en una variación porcentual de potencia respecto al incremento de altura de $0.54 \%$ por cada $100 \mathrm{~m}$ de altitud.

La variación de potencia en las cocinas, producto de la variación de la presión atmosférica a medida que se incrementa la altitud sobre el nivel mar, no se contempló por los fabricantes en el diseño de las cocinas anafes comerciales. Debido a que operando a GLP se observan la disminución por ejemplo de $1.17 \mathrm{~kW}$ a $0.86 \mathrm{~kW}$ como es en el caso de la cocina CA-01, además la cocina pierde su condición de quemador semirapido como señalan las normas técnicas de Ecuador y Chile.

Por otro lado, mediante el incremento del diámetro del inyector se compensa la perdida de potencia, es así como la cocina prototipo con un inyector de $0.7 \mathrm{~mm}$ a una presión de 28 mbar compensa la perdida de potencia y opera con una potencia de $1.5 \mathrm{~kW}$, como sería normalmente a una altitud como la de Lima, a una altitud de $4155 \mathrm{msnm}$. 
La disminución de potencia con el incremento de la altitud, permite que el rendimiento térmico se incremente como se registró en todas las cocinas anafes ensayados. Para una altitud por encima de los 2000 msnm la cocina prototipo utilizando GLP con un inyector de $0.7 \mathrm{~mm}$ y una presión de 28 mbar tiene un rendimiento térmico superior a $50 \%$ superando de esta manera a 3 de las 4 cocinas comerciales evaluadas.

Las emisiones cambian con el incremento de la altitud. Lo que nos lleva a concluir que su diseño y fabricación de las cocinas anafes comerciales no se ajustan para tener bajas emisiones en el rango de altitud trabajado.

Se concluye finalmente que una quemador atmosférico domestico cumplirá satisfactoriamente respecto a tiempo, ahorro y salud si tiene una potencia entre $2 \mathrm{~kW}$ y $1.4 \mathrm{~kW}$, un rendimiento térmico mayor a $50 \%$ y emisiones por debajo de $0.15 \%(C O)_{N}$ por encima de los $2200 \mathrm{msnm}$.

\section{AGRADECIMIENTOS}

Se agradece a INNOVATE PERU que a través de los Proyectos Concursables de Investigación Básica y Aplicada 157-FINCYT-IA-2013 en cooperación con la Pontificia Universidad Católica del Perú financiaron la investigación.

\section{REFERENCIAS}

Aggarwal, R.K., Chandel, S.S., Review of improved cookstoves programme in Western Himalayan State of India, Biomass and Bioenergy, 27, 131-144 (2004)

Amell, A., Agudelo, J., y Cortés, J., Verificación experimental del efecto de la altitud sobre la potencia térmica de un quemador atmosférico, Revista Facultad de Ingeniería, 25, 26-35 (2016)

Baukal, Ch., Industrial Burners Handbook, Nueva York, CRC Press LLC (2003)

Brucart, Enrique-Borras, Gas Natural: Características, distribución y aplicaciones industriales, Reverte, Barcelona-España (1987)

Cengel, Y.A. y M. Boles Termodinámica, McGraw-Hill, (México (2003)

Dirección General de Electricidad Ministerio de Energía y Minas. Guía N¹. Elaboración de Proyectos de Guías de Orientación del Uso Eficiente de la Energía y de Diagnóstico Energético Sector Residencial (2008)

Fulford, D., Biogas Stove Design, Reading, U.K., Kingdom Bioenergy Ltd. (1996)

Fuller, R.J., Zahnd, A. y Thakuri, S., Improving comfort levels in a traditional high altitude Nepali house, Building and Environment, 44, 479-489 (2009)

Jones, H.R.N. Domestic Gas Burber Design, Taylor \& Francis Library (2005)

INN-CHILE, Artefactos de uso doméstico para cocinar, que se utilizan combustibles gaseosos - Parte 1: Requisitos generales de fabricación y métodos de ensayo, Santiago, Modificada (2001)

Iral, L., Amell, A., Performance study of an induced air porous radiant burner for household applications at high altitude, Applied Thermal Engineering, 83, 31-39 (2015)

Namkhat, A. y Jugjai, S., The Effect of Primary Air Preheat on the Primary Aeration of a Self- aspirating Burner, The First TSME International Conference on Mechanical Engineering (2010)

Olubiyi, O., Iheanyichukwu, A., Dauda M. y Olukayode, F., Design and Construction of a Biogas Burner, Departamento de Ingeniería Mecánica, Ahmadu Bello University (2014)

OSINERGMIN, El gas natural y sus diferencias con el GLP (2012)

Pantangi, V.K., Mishra, S.C., Muthukumar, P., Reddy R., Studies on porous radiant burners for LPG (liquefied petroleum gas) cooking applications (2011) 
Rojas, F., F. Jiménez, A. Álvarez, y R. Ríos, Análisis de Mezcladores Aire-Gas combustible en un quemador de premezcla doméstico, $12^{\circ}$ Congreso Iberoamericano de Ingeniería Mecánica, 893-900 (2014)

Surange, J., Patil, N. y Rajput, A., Performance Analysis of Burners used in LPG Cooking Stove-A Review, International Journal of Innovative Research in Science, Engineering and Technology, ISSN (online) 23198753, Vol. 3 (2014)

Zhou, Y., Huang X. y Li L., Comparative study on the combustion characteristics of an atmospheric induction stove in the plateau and plain regions of China, Applied Thermal Engineering, 111, $301-307$ (2016) 